ARTIGO ORIGINAL

\title{
Produtos processados e ultraprocessados e ingestão de nutrientes em crianças
}

\author{
Processed and ultra-processed food products and nutrient intake in children
}

\author{
Giovanna Tedesco Barcelos ${ }^{1}$, Fernanda Rauber ${ }^{2}$, Márcia Regina Vitolo ${ }^{3}$
}

\section{RESUMO}

Objetivo: Avaliar a ingestão de produtos alimentícios processados e ultraprocessados entre crianças de baixa condição socioeconômica e sua influência na ingestão de energia, macronutrientes, sódio e fibras.

Materiais e Métodos: Estudo transversal com dados de crianças de 7-8 anos que participaram de um ensaio de campo randomizado realizado em São Leopoldo/RS. Foram coletados dados socioeconômicos e familiares e realizados dois inquéritos recordatórios de 24 horas. A avaliação do consumo de produtos alimentícios pelas crianças foi obtida por meio da classificação dos alimentos que considera o grau de processamento utilizado na produção. A quantidade em gramas e a estimativa da energia proveniente dos produtos alimentícios foram obtidas e os tercis do percentual de energia total da dieta proveniente desses produtos foram utilizados nas análises.

Resultados: Foram avaliados os dados de 307 crianças. A média de energia da dieta proveniente de produtos alimentícios processados e ultraprocessados representou $48,6 \%$ da energia total consumida pelas crianças. Entre as crianças no maior tercil de percentual de energia da dieta proveniente destes produtos alimentícios, o consumo de energia, carboidratos, gorduras totais, saturadas e sódio foi maior que nos demais tercis, enquanto o consumo de proteínas e fibras foi menor $(\mathrm{p}<0,05)$.

Conclusão: Entre crianças escolares de baixa condição socioeconômica, o consumo de produtos alimentícios processados e ultraprocessados representaram aproximadamente $50 \%$ da energia diária consumida, sugerindo risco para desenvolvimento de obesidade e doenças associadas visto que o consumo desses produtos foi associado a dietas com mais energia, gorduras e sódio e menor teor de proteínas e fibras.

Palavras-chave: alimentos industrializados; crianças; ingestão de energia.

\begin{abstract}
Objective: To assess the consumption of processed and ultra-processed food products among children of low socioeconomic status and its influence on energy, macronutrient, sodium and fiber intake.

Materials and Methods: This cross-sectional study included data from children aged 7-8 who participated in a randomized field trial conducted in São Leopoldo, Brazil. Socio-economic and family data were collected and two 24hour dietary recalls were conducted. The children's consumption of processed food products was evaluated by classifying the foods by the degree of processing used in their production. The amounts in grams and estimated energy of the food products were obtained and the tertiles of the percentage of total dietary energy coming from these products were used in the analyses.

Results: Data from 307 children were evaluated. The average dietary energy provided by the food products was $48.6 \%$ of the total energy consumed by the children. Among the children in the highest tertile of energy coming from food products, energy, carbohydrate, total fat, saturated fat and sodium intake was higher than in the other tertiles while protein and fiber intake was lower $(p<0.05)$.

Conclusions: Among schoolchildren of low socio-economic status, processed and ultra-processed food product consumption represented approximately $50 \%$ of daily energy intake, suggesting a risk for developing obesity and related diseases as the consumption of these products has been linked to diets higher in energy, fat and sodium and lower in protein and fiber.
\end{abstract}

Keywords: industrialized food; child; energy intake.

\footnotetext{
${ }^{1}$ Nutricionista. Universidade Federal de Ciências da Saúde de Porto Alegre, RS, Brasil.

${ }^{2}$ Nutricionista. Mestre em Ciências da Saúde. Programa de Pós-Graduação em Ciências da Saúde, Universidade Federal de Ciências da Saúde de Porto Alegre, RS, Brasil.

${ }^{3}$ Nutricionista. Doutora em Ciências Biológicas. Departamento de Nutrição, Universidade Federal de Ciências da Saúde de Porto Alegre, RS, Brasil.
} 


\section{INTRODUÇÃO}

A evolução do padrão alimentar do brasileiro nas últimas décadas mostra substituição do consumo de alimentos in natura e minimamente processados, tais como arroz, feijão e farinha de mandioca, por alimentos altamente processados e ultraprocessados, como pães, refrigerantes, biscoitos, carnes processadas e refeições prontas ${ }^{1}$. Estas mudanças na alimentação da população podem estar associadas ao aumento da ingestão de açúcar de adição, sódio, gordura saturada e trans e à diminuição do consumo de fibras ${ }^{2}$, padrão alimentar de risco para deficiências nutricionais, excesso de peso e doenças crônicas relacionadas ${ }^{3}$.

Os produtos processados são alimentos in natura que foram adicionados de sal, óleo ou açúcar, enquanto os ultraprocessados são produtos essencialmente industriais, feitos a partir de substâncias derivadas de alimentos ${ }^{4}$. Estudos sobre os hábitos alimentares de escolares, realizados no Brasil e em outros países, evidenciam consumo frequente e elevado de produtos ultraprocessados ricos em açúcares e gorduras, com destaque para refrigerantes ${ }^{5,6}$, fast food $^{7,8}$, salgadinhos ${ }^{9}$ e biscoitos ${ }^{10}$. Esses produtos possuem características sensoriais que facilitam e estimulam a prática de comer entre as refeições e o consumo excessivo de energia $^{11,12}$. Além disso, as estratégias agressivas de marketing promovidas por esses produtos ${ }^{13}$ e 0 ambiente em que o escolar está inserido ${ }^{7,8}$ favorecem o maior consumo desses produtos alimentícios. Foi descrito que $48 \%$ dos anúncios de alimentos veiculados em propagandas da televisão durante a programação infantil eram de produtos ricos em açúcares e gorduras ${ }^{14}$ e que em três escolas públicas na cidade de São Paulo os alunos tinham acesso facilitado de produtos alimentícios processados e ultraprocessados nos comércios locais e próximos às escolas ${ }^{15}$.

Concomitantemente ao padrão alimentar do escolar caracterizado pelo consumo elevado de produtos industrializados e altamente processados, a prevalência de sobrepeso e obesidade entre crianças ao longo dos anos aumentou drasticamente ${ }^{16,17}$. No Brasil, a Pesquisa de Orçamento Familiar de 2008-2009 constatou prevalência de excesso de peso de $33,5 \%$ e de obesidade de $14 \%$ entre crianças de 5 a 9 anos de idade $^{16}$. Esses dados são preocupantes, considerandose que o estado nutricional e o padrão alimentar adquiridos na infância tendem a permanecer na vida adulta $^{18,19}$.

Até o momento não foram identificados estudos que tenham relacionado o consumo de produtos alimentícios processados e ultraprocessados com a ingestão de nutrientes em crianças. Assim, objetivou-se avaliar a influência desses produtos alimentícios na dieta de crianças em idade escolar de baixo nível socioeconômico na ingestão de energia, macronutrientes, sódio e fibras.

\section{MATERIAIS E MÉTODOS}

Estudo transversal conduzido com dados de crianças em idade escolar que participaram de um ensaio de campo randomizado de aconselhamento dietético sobre aleitamento e práticas alimentares durante 0 primeiro ano de vida ${ }^{20}$. Quinhentos pares mãe-bebê foram recrutados, entre outubro de 2001 e junho de 2002, na maternidade do Sistema Único de Saúde do Hospital Centenário na cidade de São Leopoldo/RS. Os critérios de inclusão do estudo maior foram nascimento a termo ( $\geq 37$ semanas) e peso de nascimento adequado $(\geq 2500 \mathrm{~g})$. Os critérios de exclusão foram mães HIV positivo, crianças com má-formação congênita e em unidade de tratamento intensivo. Para a presente análise foi considerado como critério de inclusão possuir os dados dietéticos completos. As coletas de dados foram realizadas por estudantes de graduação em nutrição por meio de visitas domiciliares, sendo que os dados socioeconômicos das famílias foram coletados nas etapas iniciais do estudo maior.

Todos os procedimentos do estudo foram aprovados pelo Comitê de Ética da Universidade Federal de Ciências da Saúde de Porto Alegre, sob o processo número 075/05, e o termo de consentimento foi obtido de todas as mães que aceitaram participar da pesquisa. Para o presente estudo, as análises foram realizadas agrupando-se os grupos intervenção e controle do estudo maior.

\section{Coleta de dados dietéticos}

Aos 7 e 8 anos de idade das crianças realizou-se a 
TABELA 1 - Sistema de classificação NOVA ${ }^{21}$.

\section{Classificação}

Exemplos

\section{Grupo 1 - Alimentos in natura ou minimamente processados}

Alimentos in natura incluem todos os alimentos na sua forma animal ou vegetal de origem. Os alimentos minimamente processados são aqueles que foram alterados, mas sem que nenhum ingrediente fosse adicionado.

\section{Grupo 2 - Ingredientes culinários processados}

São constituintes dos alimentos in natura. Os métodos de processamentos incluem: prensagem, moagem, refinação, pulverização, entre outros.

\section{Grupo 3 - Produtos alimentícios}

\section{Produtos Processados}

Os ingredientes a eles adicionados tem a finalidade de torná-los mais duráveis e palatáveis. São produzidos para serem consumidos como parte de refeições ou como lanches. Normalmente são hiperpalatáveis.

\section{Produtos Ultraprocessados}

São produzidos a partir de ingredientes, que em sua maioria não se encontram em supermercados. São duráveis, ultrapalatáveis e rentáveis.

Os métodos de processamentos incluem: hidrogenação, hidrólise, précozimento ou pré-fritura e modificação de amidos, entre outros.
Frutas, verduras, carnes e ovos frescos.

Alimentos in natura congelados ou embalados a vácuo. Leite fresco, pasteurizado ou fermentado.

Café, chá, sucos $100 \%$ não adoçados e água pura.

Óleos vegetais, gorduras animais, açúcar, sal, farinhas, amido e massas sem pré-cozimento.

Conservas de legumes e compotas de fruta (ex: frutas em calda).

Atum ou sardinha em óleo, carne ou peixe defumados, presunto, bacon e queijos.

Cereais matinais, misturas para bolo, barras de cereal, sopas e massas instantâneas, batatas chips, salgadinhos, hamburguer, cachorro quente, carne empanada tipo nuggets, molhos, pães, biscoitos, sorvetes, margarinas, fórmulas infantis, refrigerantes, pizza, pratos compostos por carnes ou vegetais prontos ou pré-prontos para consumo.

Adaptado de Monteiro et al. ${ }^{4}$ (2012).

coleta de dados dietéticos por meio de dois inquéritos recordatórios de 24 horas realizados com a mãe e com a criança em dias não consecutivos e utilizando a técnica dos múltiplos passos. As mães e as crianças foram questionadas sobre todos os alimentos e bebidas consumidos pela criança no dia anterior, com detalhes sobre os tipos de alimentos, quantidades, marcas e métodos de preparação. Uma revisão detalhada de todos os alimentos relatados foi realizada ao final da entrevista para a correção de algum dado errôneo ou esquecido. As porções consumidas foram observadas com o auxílio de um álbum de fotos de utensílios e alimentos que foi elaborado especialmente para a pesquisa. $O$ cálculo nutricional da ingestão alimentar foi realizado utilizando o programa Nutwin versão 1.5 (Universidade Federal de São Paulo, Brasil) ampliada com a adição de alimentos disponíveis por tabelas de composição química de alimentos e aqueles fornecidos pelas indústrias brasileiras. A coleta de dados não incluiu o sal adicionado durante o cozimento e/ou a mesa, nem a ingestão de sódio proveniente de água pura.

Os itens presentes nos inquéritos alimentares foram classificados segundo o método proposto por Monteiro et al. $^{4}$ - recentemente classificado como sistema de classificação $N V^{21}$ - que é baseado na extensão e propósito do processamento de alimentos. Essa classificação agrupa os itens de consumo em três grupos: 1) Alimentos in natura ou minimamente processados; 2) Ingredientes culinários processados; e 3) Produtos alimentícios processados e ultraprocessados. A tabela 1 mostra as definições e os exemplos dos itens de consumo alimentar de cada grupo. Para este estudo, apenas os alimentos do grupo 3 (Produtos alimentícios processados e ultraprocessados) foram avaliados, sendo quantificados a quantidade em gramas consumida pelas crianças e o percentual de energia provenientes desses produtos alimentícios em relação à energia total da dieta.

Os dados foram submetidos à dupla digitação e 
validados no Programa Epi Info versão 6,4 (Centers for Disease Control and Prevention, EUA) e as análises estatísticas foram realizadas no Programa SPSS versão 16,0 (Statistical Package for Social Sciences, SPSS Inc., EUA). Análises de frequência foram realizadas para descrição das variáveis categóricas. Utilizou-se a análise de variância (ANOVA) de uma via e as comparações múltiplas de Bonferroni para identificar as diferenças entre as variáveis de consumo de acordo com os grupos. Foi considerado, neste estudo, como nível de significância valor de $p<0,05$.

\section{RESULTADOS}

Das 307 crianças analisadas, 57\% eram do sexo masculino. A maior parte das famílias (70\%) possuía renda de até três salários mínimos e $56,2 \%$ das mães tinham menos de oito anos de escolaridade (tabela 2). A média de energia da dieta proveniente de produtos alimentícios foi de 776,15kcal, o que representou, em média, $48,6 \%$ da energia total consumida pelas crianças. Não houve diferença estatística na média de energia proveniente desses produtos entre sexos ( $48,8 \%$ entre os meninos e $48,4 \%$ entre as meninas).

A análise de ingestão de nutrientes de acordo com os tercis de energia proveniente de produtos alimentícios processados e ultraprocessados está apresentada na tabela 3. Entre as crianças no maior tercil de percentual de energia da dieta proveniente de produtos alimentícios processados e ultraprocessados, a ingestão de energia, carboidratos, gorduras totais, gorduras saturadas e sódio foi maior do que dos demais tercis, enquanto o consumo de proteínas e fibras foi menor $(p<0,05)$. Não houve diferença estatística no consumo de colesterol pelas crianças segundo os tercis de energia proveniente de produtos alimentícios processados e ultraprocessados.

\section{DISCUSSÃo}

Os resultados deste estudo mostraram que, entre crianças escolares de baixa condição socioeconômica, o consumo de produtos alimentícios processados e ultraprocessados representou $48 \%$ da energia diária consumida. Além disso, o consumo desses produtos foi associado às dietas mais energéticas, com maiores
TABELA 2 - Características das crianças e das famílias $(n=307)$.

\begin{tabular}{lr}
\hline Variável & $\mathbf{n}(\%)$ \\
\hline Sexo & \\
$\quad$ Masculino & $175(57)$ \\
$\quad$ Feminino & $132(43)$ \\
Renda Familiar (salários mínimos) & \\
$\quad<3$ salários mínimos & $210(70)$ \\
$\geq 3$ salários mínimos & $90(30)$ \\
Idade da mãe ao nascimento da criança & \\
$\quad<20$ anos & $57(18,6)$ \\
$\geq 20$ anos & $250(81,4)$ \\
Escolaridade da mãe & \\
$\quad<8$ anos & $172(56,2)$ \\
$\quad \geq 8$ anos & $134(43,8)$ \\
Ocupação da Mãe & \\
Empregada & $134(43,8)$ \\
Desempregada & $194(65,1)$ \\
\hline
\end{tabular}

O número amostral variou de acordo com a disponibilidade dos dados.

teores de carboidratos, gorduras e sódio e, ainda, com menor ingestão de proteínas e fibras. Essas características alimentares são consideradas fatores de risco para excesso de peso ${ }^{22-24}$, principalmente quando consumidos em substituição aos alimentos in natura, como verduras e legumes ${ }^{25}$. O aumento do consumo desses produtos pela população, como evidenciado por pesquisa nacional ${ }^{17}$, pode ser uma das explicações para a tendência crescente da prevalência de excesso de peso, principalmente entre crianças. O percentual de crianças com excesso de peso aumentou de $10 \%$ em 1974-1975 para 33\% em 2008-2009 no Brasil, enquanto a prevalência de obesidade aumentou cinco vezes no mesmo período ${ }^{1}$.

Poucos estudos têm avaliado a participação de produtos alimentícios processados e ultraprocessados na adequação da alimentação. Recente estudo realizado com amostra representativa da população brasileira demonstrou associação positiva e independente entre a disponibilidade domiciliar de produtos ultraprocessados e a obesidade ${ }^{26}$. Tavares et al. $^{27}$, em estudo transversal realizado com adolescente de Niterói/RJ, encontraram que o consumo de produtos alimentícios processados e ultraprocessados foi associado à síndrome metabólica. No entanto, ambos estudos citados não avaliaram a relação do consumo de 
TABELA 3 - Análise do consumo de energia e nutrientes por crianças em idade escolar de acordo com os tercis do percentual de energia total da dieta proveniente de produtos alimentícios (processados e ultraprocessados). $\mathrm{n}=307$.

\begin{tabular}{|c|c|c|c|}
\hline & \multicolumn{3}{|c|}{ Percentual de energia total da dieta proveniente de produtos alimentícios } \\
\hline & $1^{\circ}$ tercil & 2o tercil & 3ำtercil \\
\hline & $(\leq 42,3 \%)$ & $(\geq 42,4 \%$ e $\leq 54,4 \%)$ & $(\geq 54,5 \%)$ \\
\hline & média $\pm \mathrm{DP}$ & média $\pm D P$ & média $\pm D P$ \\
\hline Energia (kcal) & $1475,5 \pm 369,5$ & $1527,1 \pm 307,0$ & $1675,2 \pm 376,7^{\S}$ \\
\hline Carboidratos (g) & $208,4 \pm 55,69$ & $219,5 \pm 43,4$ & $241,2 \pm 54,2^{\S}$ \\
\hline Proteínas (g) & $59,3 \pm 16,9^{*}$ & $55,5 \pm 14,9$ & $53,2 \pm 15,2$ \\
\hline Lipídeos (g) & $46,2 \pm 17,4$ & $49,1 \pm 14,2$ & $56,5 \pm 17,8^{\S}$ \\
\hline Gordura saturada (g) & $14,0 \pm 5,6$ & $14,7 \pm 5,3$ & $16,9 \pm 5,9^{\S}$ \\
\hline Colesterol (mg) & $160,7 \pm 66,4$ & $147,7 \pm 70,16$ & $141,9 \pm 73,9$ \\
\hline Sódio (mg) & $1231,5 \pm 536,4$ & $1456,2 \pm 495,0^{\pi}$ & $1806,0 \pm 675,2^{\S}$ \\
\hline Fibras (g) & $12,22 \pm 4,59$ & $10,89 \pm 4,26$ & $9,68 \pm 4,67^{\epsilon}$ \\
\hline
\end{tabular}

ANOVA com correção de Bonferroni;

I Significativamente maior que o $1^{\circ}$ tercil $(p<0,05)$;

$€$ Significativamente menor que o $1^{\circ}$ e $2^{\circ}$ tercis $(p<0,05)$;

$¥$ Significativamente maior que o $3^{\circ}$ tercil $(p<0,05)$;

$\S$ Significativamente maior que $01^{\circ}$ e $2^{\circ}$ tercis $(p<0,05)$.

produtos alimentícios processados e ultraprocessados e o impacto no consumo de nutrientes. Outros estudos que avaliaram o consumo de certos tipos de produtos alimentícios que se enquadram na classificação de processados e ultraprocessados, tais como, pizza, refrigerante, cachorro quente, suco industrializado e doces, mostraram que esses são os itens de consumo alimentar que mais contribuíram para energia total ${ }^{28,29}$, teores de gorduras e açúcares na dieta de crianças ${ }^{5,9,30}$ e, ainda, foram associados ao excesso de peso ${ }^{23}$. Além disso, os resultados desse estudo mostraram que a maior participação de produtos alimentícios processados e ultraprocessados na alimentação foi associada a menor ingestão de fibra pelas crianças, sugerindo risco aumentado para o desenvolvimento de obesidade e doenças crônicas relacionadas ${ }^{31}$, além de constipação ${ }^{32}$.

O menor consumo de proteínas pelas crianças que apresentavam maior participação de produtos alimentícios processados e ultraprocessados na alimentação pode ser reflexo da substituição dos alimentos básicos fontes de proteínas, como o feijão e carnes in natura, por esses produtos como já evidenciado anteriormente ${ }^{1,33}$. Assim, o consumo elevado de produtos alimentícios processados e ultraprocessados aumenta o percentual de gorduras e carboidratos da dieta em detrimento do percentual de proteínas.

Destaca-se que o consumo de sódio apresentou aumento mais acentuado do que os demais nutrientes à medida que o percentual de energia da dieta provinda de produtos alimentícios processados e ultraprocessados aumentou entre as crianças. Vitolo et al. $^{34}$, em estudo realizado com a mesma amostra de crianças em idade pré-escolar, observaram que as principais fontes de sódio na alimentação foram o macarrão instantâneo, a batata chips e os pães produtos considerados ultraprocessados; e que o consumo de sódio acima das recomendações dietéticas foi associado à pressão sistólica elevada já na infância, expondo essas crianças ao risco de desenvolvimento de hipertensão arterial precoce e/ou futuramente. Enfatizase que este estudo não contabilizou o sal utilizado nas preparações, reforçando, portanto, a contribuição dos produtos alimentícios processados e ultraprocessados na elevada ingestão de sódio pelas crianças.

Os resultados referentes ao colesterol dietético demonstram que o consumo desse nutriente não apresentou diferença entre os tercis de participação dos produtos alimentícios processados e ultraprocessados no consumo alimentar das crianças. Uma possível justificativa para esse achado é que o consumo de colesterol pelas crianças estava dentro dos limites considerados adequados já que estudos prévios 
realizados com a mesma amostra mostraram que mais de $90 \%$ das crianças apresentaram consumo de colesterol compatível com as recomendações nutricionais $^{35,36}$. Além disso, destaca-se que as principais fontes de colesterol na dieta são alimentos de origem animal, como ovos, laticínios, vísceras de animais e frutos do mar e que produtos alimentícios ultraprocessados, frequentemente consumidos por crianças, como bolachas recheadas, salgadinhos chips e outras guloseimas, são ricos em gorduras, porém com baixo teor de colesterol ${ }^{37,38}$.

Entre as limitações desse estudo, destaca-se que os resultados não podem ser generalizados para a população geral, pois foram incluídas apenas crianças de baixa condição socioeconômica. Os dados da alimentação das crianças foram reportados pelas mães e pelos filhos estando sujeitos a erros de notificação. Contudo, utilizou-se a técnica dos múltiplos passos para facilitar a coleta dos dados dietéticos por meio do recordatório alimentar de 24 horas das crianças, minimizando, assim, os possíveis erros de relato.

Este estudo mostrou que, entre crianças escolares de baixa condição socioeconômica, o consumo de produtos alimentícios processados e ultraprocessados representou aproximadamente $50 \%$ da energia diária consumida, sugerindo risco para desenvolvimento de obesidade e doenças associadas visto que o consumo desses produtos foi associado a dietas com mais energia, gorduras e sódio e menor teor de proteínas e fibras. Esses resultados podem ser ainda mais relevantes ao considerarmos que os hábitos alimentares desenvolvidos na infância tendem a permanecer na vida adulta, influenciando as condições de saúde em curto e longo prazo. Programas que visem à promoção da saúde do escolar, envolvendo a educação alimentar para as famílias, devem priorizar a redução da ingestão de produtos alimentícios processados e ultraprocessados, principalmente em substituição aos alimentos in natura e minimamente processados fontes de vitaminas e nutrientes.

\section{REFERÊNCIAS}

1. Instituto Brasileiro de Geografia e Estatística. Pesquisa de Orçamentos Familiares 2008-2009. Avaliação nutricional da disponibilidade domiciliar de alimentos no Brasil. Rio de Janeiro (RJ): IBGE; 2010.

2. Monteiro CA, Levy RB, Claro RM, Castro INRR, Cannon G. Increasing consumption of ultra-processed foods and likely impact on human health: evidence from Brazil. Public Health Nutrition. 2010; 14(1), 5-13. doi:10.1017/ S1368980010003241

3. Kavey R-EW, Simons-Morton DG, de Jesus JM, Suppl Editors. National Heart, Lung, and Blood Institute. Expert panel on inte-grated guidelines for cardiovascular health and risk reduction in children and adolescents: summary report. Pediatrics. 2011;128 Suppl 5:S213-56. doi: 10.1542/peds.2009-2107C.

4. Monteiro CA, Cannon G, Levy RB, Claro R, Moubarac J-C. The Food System. Ultra-processing. The big issue for nutrition, disease, health, well-being. World Nutrition, December 2012; 3(12):527-69.

5. Carmo MB, Toral N, Silva MV, Slater B. Consumo de doces, refrigerantes e bebidas com adição de açúcar entre adolescentes da rede pública de ensino de Piracicaba, São Paulo. Rev Bras de Epidemiol. 2006; 9(1): 121-30.

6. Rivera FSR, Souza EMT. Consumo alimentar de escolares de uma comunidade rural. Comun Ciênc Saúde. 2006;17(2): 111-9.

7. Babey $\mathrm{SH}$, Wolstein J, Diamant AL. Food Environments Near Home and School Related to Consumption of Soda and Fast Food. Los Angeles, CA: UCLA Center for Health Policy Research; 2011.

8. Langellier BA. The food environment and student weight status, Los Angeles County, 2008-2009. Prev Chronic Dis. 2012; 9:110191.

9. Ford CN, Slining MM, Popkin BM. Trends in dietary intake among US 2 to 6-year-old children, 1989-2008. J Acad Nutr Diet. 2013; 113(1):35-42. doi: 10.1016/ j.jand.2012.08.022.

10. Johansson I, Holgerson PL, Kressin NR, Nunn ME, Tanner AC. Snacking habits and caries in young children. Caries Res. 2010; 44:421-30. doi: 10.1159/000318569

11. Ifland JR, Preuss HG, Marcus MT, Rourke KM, Taylor WC, Burau K, Jacobs WS, Kadish W, Manso G. Refined food addiction: A classic substance use disorder. Med Hypotheses. 2009; 72(5):518-26.

12. Yeomans MR, Blundell JE, Leshem M. Palatability: response to nutritional need or need-free stimulation of appetite? Brit J Nutr. 2007; 92 Suppl 1:3-14. doi: 10.1079/BJN20041134.

13. Mehta K, Phillips C, Ward P, Coveney J, Handsley E, Carter P. Marketing foods to children through product packaging: prolific, unhealthy and misleading. Public Health Nutr. 2012; 15(9):1763-70. doi: 10.1017/ S1368980012001231.

14. Costa SMM, Horta PM, Santos LC dos. Análise dos alimentos anunciados durante a programação infantil em emissoras de canal aberto no Brasil. Rev Bras Epidemiol 2013; 16(4): 976-83.

15. Leite FH, Oliveira MA, Cremm EC, de Abreu DS, Maron LR, Martins PA. Availability of processed foods in the perimeter of public schools in urban areas. J Pediatr (Rio J). 2012; 88(4):328-34. doi:10.2223/JPED.2210.

16. de Onis M, Blössner M, Borghi E. Global prevalence and trends of overweight and obesity among preschool children. Am J Clin Nutr. 2010; 92: 1257-64. doi: 10.3945/ajcn.2010.29786.

17. Instituto Brasileiro de Geografia e Estatística. Pesquisa de Orçamentos Familiares 2008-2009. Antropometria e estado nutricional de crianças, adolescentes e adultos no Brasil. Rio de Janeiro (RF): IBGE; 2010.

18. Craigie AM, Lake AA, Kelly SA, Adamson AJ, Mathers JC. 
Tracking of obesity-related behaviours from childhood to adulthood: a systematic review. Maturitas. 2011; 70(3):266-84. doi: 10.1016/j.maturitas.2011.08.005.

19. Gardner DS, Hosking J, Metcalf BS, Jeffery AN, Voss LD, Wilkin TJ. Contribution of early weight gain to childhood overweight and metabolic health: a longitudinal study (EarlyBird 36). Pediatrics. 2009; 123: e67-73. doi: 10.1542/peds.2008-1292.

20. Vitolo MR, Bortolini GA, Feldens CA, Drachler ML. Impactos da implementação dos dez passos da alimentação saudável para crianças: ensaio de campo randomizado. Cad Saúde Pública. 2005; 21(5):1448-57. doi: 10.1590/S0102-311X2005000500018.

21. Moubarac JC, Parra DC, Cannon G, Monteiro CA. Food classification systems based on food processing: significance and implications for policies and actions: a systematic literature review and assessment. Curr Obes Rep. 2014; 3(2):256-72. doi: 10.1007/s13679-014-0092-0.

22. Lanigan J, Singhal A. Early nutrition and long-term health: a practical approach. Proc Nutr Soc. 2009 Nov;68(4):422 9. doi: 10.1017/S002966510999019X.

23. Rouhani $\mathrm{MH}$, Mirseifinezhad M, Omrani N, Esmaillzadeh A, Azadbakht L. Fast food consumption, quality of diet, and obesity among Yahanian adolescent girls. J Obes. 2012; 2012:597924. doi: 10.1155/2012/597924.

24. Song WO, Wang Y, Chung CE, Song B, Lee W, Chun OK. Is obesity development associated with dietary sugar intake in the U.S?. Nutrition. 2012; 28:1137-41. doi: 10.1016/j.nut.2012.03.008.

25. Fernandes RA, Casonatto J, Christofaro DGD, Ronque ERV, de Oliveira AR, Júnior IFF. Riscos para o excesso de peso entre adolescentes de diferentes classes socioeconômicas. Rev Assoc Med Bras. 2008; 54(4):3348.

26. Canella DS, Levy RB, Martins AP, Claro RM, Moubarac JC, Baraldi LG, Cannon G, Monteiro CA. Ultra-processed food products and obesity in brazilian households (20082009). PLoS One. 2014; 9(3):e92752. doi: 10.1371/journal.pone.0092752.

27. Tavares LF, Fonseca SC, Rosa MLG, Yokoo EM. Relationship between ultra-processed foods and metabolic syndrome in adolescents from a Brazilian Family Doctor Program. Public Health Nutrition. 2011; 15(1):82-7. doi: 10.1017/S1368980011001571.

28. De Graaf, C. Effects of snacks on energy intake: an evolutionary perspective. Appetite. 2006; 47(1):18-23. doi:10.1016/j.appet.2006.02.007.

29. Piernas C, Popkin BM. Increased portion sizes from energy-dense foods affect total energy intake at eating occasions in US children and adolescents: patterns and trends by age group and sociodemographic characteristics, 1977-2006. Am J Clin Nutr. 2011; 94:132432. doi: 10.3945/ajcn.110.008466.

30. Reedy J, Krebs-Smith, SM. Dietary sources of energy, solid fats, and added sugars among children and adolescents in the United States. J Am Diet Assoc. 2010; 110(10):1477-84. doi: 10.1016/j.jada.2010.07.010.

31. Slavin JL. Position of the American Dietetic Association: health implications of dietary fiber. J Am Diet Assoc. 2008; 108: 1716-31.

32. Mello CS, Freitas KC, Tahan S, de Morais MB. Consumo de fibra alimentar por crianças e adolescentes com constipação crônica: influência da mãe ou cuidadora e relação com excesso de peso. Rev Paul Pediatr 2010; 28(2):188-93.

33. Martins AP, Levy RB, Claro RM, Moubarac JC, Monteiro CA. Participação crescente de produtos ultraprocessados na dieta brasileira (1987-2009). Rev Saude Publica. 2013;47(4):656-65. doi: 10.1590/S0034-8910. 2013047004968.

34. Vitolo MR, Louzada MLC, Rauber F, Campagnolo PDB. Risk factors for high blood pressure in low income children aged 3-4 years. Eur J Pediatr. 2013; 172(8):1097-103. doi: 10.1007/s00431-013-2012-9.

35. Rauber F, Hoffman DJ, Vitolo MR. Diet quality from preschool to school age in Brazilian children: a 4-year followup in a randomized control study. $\mathrm{Br} \mathrm{J}$ Nutr. 2014; 111(3):499-505. doi: 10.1017/S0007114513002857.

36. Beskow CB, da Costa ML, Rauber F, Campagnolo PD, Vitolo MR. Consumo alimentar de risco para doença cardiovascular entre crianças de baixo nível socioeconômico. Nutr Pauta; 2012; 117.

37. Castro TG, Baraldi LG, Muniz PT, Cardoso MA. Dietary practices and nutritional status of 0-24-month-old children from Brazilian Amazonia. Public Health Nutr. 2009; 12(12):2335-42. doi: 10.1017/S1368980009004923.

38. Bellisle F, Rolland-Cachera MF; Kellogg Scientific Advisory Committee. Three consecutive (1993, 1995, 1997) surveys of food intake, nutritional attitudes and knowledge, and lifestyle in 1000 French children, aged 911 years. J Hum Nutr Diet. 2007; 20(3):241-51. doi: 10.1111/j.1365-277X.2007.00773.x.

\section{Endereço para correspondência:}

Fernanda Rauber

Rua Sarmento Leite $n^{\circ} 245$, Anexo I, Sala 3

Porto Alegre/RS - CEP 90050-170

Telefone: +55 5133038798

E-mail: rauber.fernanda@gmail.com 